\title{
Exploiting Distributed Cognition to Make Tacit Knowledge Explicating
}

\author{
Mingrui $\mathrm{He}^{1}$, Yongjian $\mathrm{Li}^{2}$ \\ ${ }^{1}$ School of Management and Economics of University of Electronic Science and Technology of China, Chengdu, China; ${ }^{2}$ School of \\ Management and Economics of Southwest Jiaotong University, Chengdu, China. \\ Email: mingrui0208@21cn.com; swtjlyj@sina.com.cn
}

Received September $1^{\text {st }}, 2009$; revised October $5^{\text {th }}, 2009$; accepted October $12^{\text {th }}, 2009$.

\begin{abstract}
Distributed cognition is a new development trend of cognitivism, and is also a new research field of knowledge management. The study discusses that tacit knowledge explicating activity is a distributed cognitive activity, whose success depends on interaction of each of these factors in distributed cognitive system and none of the factor could be neglected. Further, the study exploits distributed cognition to explore how to design these factors in the system so that tacit knowledge explicating can be accomplished successfully.
\end{abstract}

Keywords: Tacit Knowledge, Distributed Cognition, Tacit Knowledge Explicating

\section{Introduction}

In today's dynamic global economy, knowledge is viewed as a key strategic and competitive resource by organizations, and effective management of individual knowledge within the work place has become critical to business $[1,2]$. Growing interest in the management of knowledge within organizations has focused on the control of tacit knowledge, which can be retained within the firm as a source of possible competitive advantage $[3,4]$. The knowledge in employee's head (tacit knowledge) is accounting for $42 \%$ of organization total knowledge, by surveying the knowledge composition of Delphi Group [5]. Further, OECD's a report named The KnowledgeBased Economy has indicated that the best value to organization is tacit knowledge of individual. So how to exploit and manage tacit knowledge always is crucial to knowledge management.

With expanding of knowledge management study, more and more scholars realize that we should pay more attention to not only technique but also personal factors [6]. To grasp the essence and regulation of human cognition is indispensable to the study of knowledge management [7]. The breakthrough of modern cognitive psychology, especially the development of distributed cognition, provides new angle of view to study tacit knowledge explicating.

Firstly, this article attempts to bring some clarification to tacit knowledge. We give an overview of historical beginnings of tacit knowledge concepts. Before we dis- cuss how to transfer tacit knowledge, it's necessary to understand what means the tacit knowledge discussed in this article. Secondly, the article will inventory the basic tenets of the concept of distributed cognition, then review the current studies of tacit knowledge explicating and discuss how to disclose the cognition activity is the most essential question. In the following section, we discuss how to disclose the cognition activity based on distributed cognition. In particular, we discuss that the change of each factor in function system would cause the change of the whole system, and each factor in the system is very important for the success of tacit knowledge explicating. If we want to make the cognitive activity successful, to design each factor in the system is necessary.

\section{Tacit Knowledge}

In 1958, Michael Polanyi [8] put forward the term (tacit knowledge) in his book named Personal Knowledge, he proposes his famous epigram "we know more than we can tell": Humans can undertake a range of activities, and thus in a key sense know how to do them, without necessarily being able to provide a complete or coherent account of their actions, their reasons for undertaking them or to explain to others how to undertake them, let alone to explain the laws of physics, biology and so on that underline them.

The term has been paid close attention by many scholars from the earliest times. Robert J. Sternberg and his colleague defined the term from the view of psychology 
[9-11]. It is viewed as knowledge that generally is acquired with little support from other people or resource, as procedural in nature, and it has direct relevance to individual's goals. P. F. Drucker defined the term from the view of management [12]. Tacit knowledge can't be explained by language, only be confirmed by demonstration. The only way to study them is apperception and exercise. They root in experience and skills. And Nonaka believed that, tacit knowledge can have both technical and cognitive dimension, and it is high personalized and high situated, it includes individual thinking model, belief and mental model etc. Those models and beliefs are so deeply rooted that we are quite hard to perceive them. But when we are looking around the world, we always receive their huge impact [4]. Tacit knowledge, which is deeply rooted in action and context, can be acquired without awareness and is typically not articulated or communicated [13].

The notion of tacit knowledge is intuitively appealing and seems to be something that we all instinctively understand as the knowledge that people have in their heads, rather than knowledge that is written down and recorded [14]. However, as Day [15] notes, the "folk-psychology" notion of tacit knowledge is simplistic and leads to the expectation that tacit knowledge can easily be transferred simply by having the knowledge holder reflection and articulate the knowledge. In fact, the real tacit knowledge remains ambiguous, with researchers applying the term with a variety of meanings and characterizations [16]. So before discussing tacit knowledge explicating, the article attempts to bring up some clarification to tacit knowledge firstly.

The nature of tacit knowledge in a business context can be viewed as a continuum with structured, codified, or explicit knowledge at one extreme and unstructured, uncodified, or tacit knowledge at the other [17]. Actually, we discuss the tacit knowledge at the extreme of knowledge continuum is complete tacit knowledge, which means that people absolutely cannot perceive them, let alone explain or articulate them, such as mental models. Between one extreme of knowledge continuum and the other extreme of knowledge continuum, there is a kind of tacit knowledge which cannot be structured or codified, but people can perceive them. For instance, skilled baker can bake delicious bread, he knows he can do that, and others also know he can do that, however he cannot articulate how to do that. We discuss the tacit knowledge which he owns is a special kind of tacit knowledge. The kind of tacit knowledge usually is in form of individual skill. But only after people can perceive the skill, the skill can be fallen the kind tacit knowledge. Tacit knowledge discussed in the article is this kind of tacit knowledge. It's no meaning to discuss tacit knowledge explicating if people absolutely cannot perceive them. In fact, for business organizations this kind of tacit knowledge has more meanings.

So we describe this kind of tacit knowledge as follows, and tacit knowledge mentioned in the following text is this kind of tacit knowledge.

Tacit knowledge is difficult to be partially or totally coded by language or words in a particular situation. The definition reveals the main features of tacit knowledge. One is difficult to code the knowledge in a particular situation which means that maybe others can code it or maybe one can code it in another particular situation. Tacit knowledge is high personalized and situated, and its cost of transfer is so high. It is formed automatically by subconscious. Its forming and utilizing aren't controlled by willingness of subjective and are manifested by inspiration, skill, habit and belief, and so on. However tacit knowledge is not mysterious experience (Polanyi), it is just can not be partial or total coded by one in a particular situation.

\section{The Concept of Distributed Cognition}

Distributed cognition, which takes cognitive overview into consideration, is a new development trend of cognitivism and a new cognitive paradigm. In 1884, Dewey wrote that organisms do not deviate from environment. It's impossible to look mental activity as individual activity without any relations [18]. One's cognition should be built on interactive relationship of human and environment [19]. Hutchins explicitly defined distributed cognition as a new basic paradigm to rethink cognitive phenomena in all fields [20]. The chief theory and methodology of distributed cognition is that it emphasizes that analytical unit of design, individual in social or in some situation instead of individual who be thought that he plays cognitive activity only in his head, and functional relations among different factors in cognitive process form functional system [21].

Having learnt these methods from cognition science, anthropology, sociology and social psychology, distributed cognition holds that to know cognitive phenomena should be from functional system point of view, which is composed of individual, other individual and artifact, and so on. Those cognitive phenomena which cannot be known only from individual point of view are pinpointed in distributed cognition. It's particularly important that distributed cognition stresses interaction among individuals and technique tools in a specific cognitive activity [22]. So distributed cognition is a system made up of cognitive subjects and environment, a new analytical unit including all things in the cognitive activity [23], and an information processing of representation to inner and external [24].

Since it was born, distributed cognition has strong vitality. It not only has learnt many advantages of traditional cognition, but also has different features from traditional cognition. Firstly, distributed cognition takes all factors into consideration in cognitive activity, puts forward a new analytical unit which is built on functional relations 
among different factors participating cognitive processing together, and forms functional systems which show different representation status among different media and at the same time harmonize these media. Secondly, distributed cognition emphasizes the distribution across individuals, artifacts and internal and external representations in terms of a common language of 'representational states' and 'media', and holds that cognition can be distributed not only within individual but also in media, cultures, social and time. Thirdly, distributed cognition also stresses the influence of social substance situation to cognition process. Finally, distributed cognition notes that communication, sharing, factors (human and artifacts, etc.) depends on each other, and artifacts play important role in distributed cognition. When artifacts are used by people, cognitive residue phenomenon will appear. As long as artifacts are applied to help cognitive action, the ability being trained in the action would be remained, even the artifacts have gone, the ability is still here and can support high level thinking.

\section{Tacit Knowledge Explicating is a Cognitive Activity with Distributed Cognition}

\subsection{The Current Studies of Tacit Knowledge Explicating}

The idea about knowledge transfer was firstly formulated by Teece in 1977 [25], he thought that technique transfer can help industry accumulate valuable knowledge and impel technology diffusion, the result can reduce technology gap among different areas. With further development of knowledge-based economy, the study of tacit knowledge explicating has been put on the agenda. Tacit knowledge has been studied that it can be explicated by deep talks including analogy, story and metaphor $[4,26,27]$, can be attained and transferred by learning and informally communication among people working as technical innovation [28], and can be exploited and applied by a new applied information technology. Tacit knowledge in inter-web of organization can be distinguished by information retrieval system based on software proxy technology [29]. Cognition mapping is also a useful implement to transfer tacit knowledge [30]. Further, Zhang [30] made differential dynamic model of organization tacit knowledge and analyzed these primary controlling parameters to influent tacit knowledge diffusing. Gao [31] made a model transfer of tacit knowledge based on ontology. Liang [32] thought informal relationship network is a primary way to transfer tacit knowledge. Tang [33] thought knowledge has biological activity, and made knowledge fermentation model borrowing biology fermentation process. Of course, SECI model is the most influential in the field of tacit knowledge explicating, which was put forward by Nonaka and Takeuchi. Socialization, externalization, combination and internalization form a circle of knowledge transformation and creating.

The current studies of tacit knowledge explicating focus mainly on the methods of explicating, transfer model and technique, especially SECI model pushes up greatly the development of knowledge management theory and practice, and becomes one of important foundations of knowledge management theory [34]. However, it is a pity that SECI doesn't take cognitive psychology into account [35] when it provides a suit of analysis paradigm [36]. In recent years, cognitive psychology has been paid more and more attention to by scholars when they are studying tacit knowledge. The inner mechanism of tacit knowledge and implicit cognition has been discussed. Implicit cognition provides empirical evidence for tacit knowledge from psychology, and tacit knowledge provides theoretical basement from epistemology [37]. And cognitive structure of tacit knowledge is also discussed, which is comprised of implicit system mechanism, ingredients transferred mechanism and motivation mechanism [38]. However, most of the research works focus on the individual cognition of tacit and few discuss on the cognitive activity of tacit knowledge explicating. From the most fundamental terms, tacit knowledge explicating is one kind of cognitive activity. The success of tacit knowledge explicating depends on individual, artifacts, environment, cultures, etc. in the cognitive activity. How to reveal the cognition activity is the most essential question. Only after having discussed the activity which is like black box, we can made further research on how to increase the efficiency of tacit knowledge explicating.

\subsection{Tacit Knowledge Explicating As Distributed Cognition}

Tacit knowledge explicating activity can fall into two forms.

One is that individual with tacit knowledge can explicate the knowledge by his own explicit knowledge and artifacts, and it can be shown as Figure 1. In this form of activity, cognition is distributed within individual, among artifacts, in culture, in environment, through time and so on.

Another is that when individual with tacit knowledge communicate with others, his tacit knowledge can be explicated by others' explicit knowledge and artifacts, and it can be shown as Figure 2. In this form of activity, cognition is distributed within individual, among individuals, among artifacts, in culture, in environment, through time and so on. The second form is a general form for tacit knowledge explicating, so we discuss the second form in this article.

When cognition is considered as a distributed system, it opens up the process of tacit knowledge explicating to inspection. This is important for tacit knowledge explicating because inspection permits people to examine the 


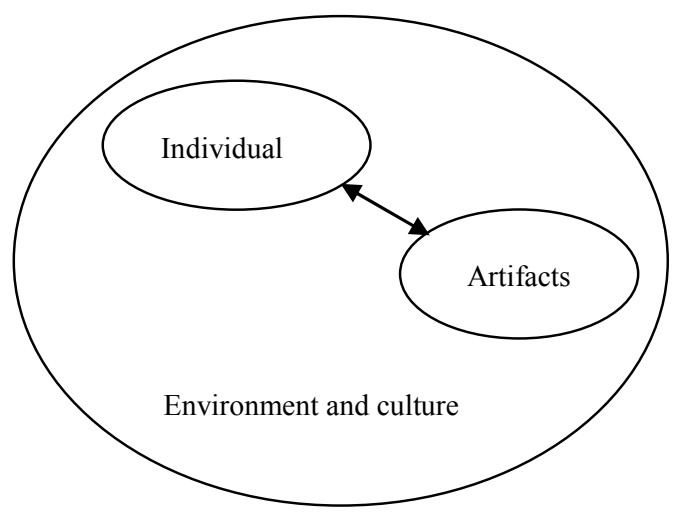

Figure 1. Form 1

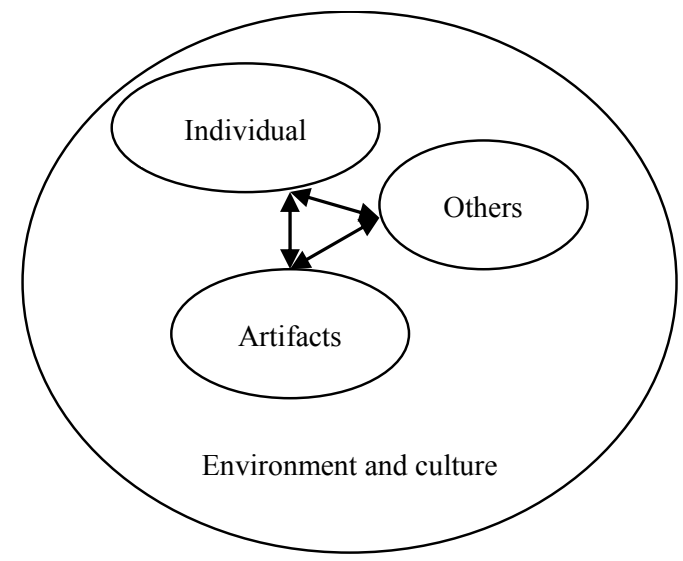

Figure 2. Form 2 variables involved in the activity and more importantly, distributed cognition, as a perspective with which to think, has the potential to enhance the likelihood of careful selections of tools by people for the activity.

In the activity, cognition is distributed:

1) Within individual: Cognition structure of individual is psychological basis to make tacit knowledge explicit;

2) Among individuals: For instance, storytelling is often thought as an effective way to make tacit knowledge explicit;

3) Among artifacts: Artifact is a core term in distributed cognition, which means tool, thinking and method and so on. When artifacts are applied, people's intelligence can be extended and people can be more intelligent and more effective. For instance, computers can make us simulate real world better, and symbols can make us express our thoughts and ideas better. Human inner cognitive ability and external artifacts together can greatly improve cognitive level [21];

4) In culture: Different individuals in the activity maybe are from different cultures, which causes they have different cognitive styles. Culture can be experienced by communicating face-to-face, and influents cognitive process indirectly. The human mind is more than the sum of localized (interiorized) cognition; our thoughts, capabilities, and actions are continuously shaped by, and co-evolve with, elements of the external world and the cultural contexts in which we operate [39];

5) In environment: Interaction of internal and external representations is influenced by environment. Any activity cannot be divorced from environment, including tacit knowledge explicating;

6) Through time: The products of earlier events can transform the nature of later events [20].

\section{Analyzing Tacit Knowledge Explicating Activity Based on Distributed Cognition}

When cognition is seen as distributed system, it has provided a very good method and angle of view to study the essence of tacit knowledge explicating which is like black box. During the cognitive activity, not only the individual with tacit knowledge should be paid attention to, but also other variables in this cognitive activity should be paid attention to [40], for instance, the cognitive state of others, the actions of others when they accept tacit knowledge, the characters of artifacts, culture state and environment state, and so on. The whole tacit knowledge explicating is a dynamic activity in which dynamic exchange is between individuals, between artifacts, between individual and artifact, etc. Of course, dynamic exchange is also in particular culture and environment. In the whole activity, the source of tacit knowledge is often considered as teacher, and the focal-point of tacit knowledge is often considered as learner.

The whole tacit knowledge explicating activity has cognitive distribution, which distribute within individual, among individuals, among artifacts, in culture, in environment and so on. Further, individuals, artifacts, culture and environment constitute a functional system. According to the functional system, the representation, transmission, and progress can be analyzed as follows by four analysis phases $[41,42]$. The method pays attention to collect raw data not only from different metaphysical and material levels, but also from the changes of representation state in the activity. So it can show the crucial moment which is maybe unclear and maybe neglected by traditional analysis, and can definitely reveal that the problem is caused by mutual influence of various factors.

In the first phase: Individual takes inner representation and forms some knowledge (for instance, individual skill) in his working memory system. He cannot express this knowledge (tacit knowledge) by any language or words, only could teach others by demonstrating over and over or using suitable artifacts. On one hand, learner undergoes instructions himself from the knowledge holder, on the other hand, he selects suitable artifacts to help himself learn it better, for instance, he can use video or audio to record situation. The records must be very detailed. To omit any subtle corner, color and odor can cause some significant information missing (be missed). In this phase, 
inner representation, interaction among artifacts and social interaction between different inner representations are primary.

In the second phase: Learner always has different cognitive structures and knowledge structures from the knowledge holder. By observing the holder and using artifacts, he could translate all information representation by video or audio records into printing press representation, including language and other words. In this phase, the interaction of inner representation state and technique tool representation is primary.

In the third phase: Learner and his (her) partners would find significant behaviors and events from the printing press representation attained in the second phase. So the continuous behavior flow is divided into lots of significant blocks.

In the fourth phase: to explain these significant behaviors and events confirmed in the third phase, then translate into corresponding theory. So the result of explicating would be attained.

When we analyze tacit knowledge explicating by the method, we need to descript all aspects of behaviors and interactions, which are so trivial and apt to take for granted, and cannot neglect the important function of environment, culture and artifacts in the cognitive activity. Different from those methods which only care the main element causing problem, the method definitively reveal a problem caused by various factors [43]. By the method to analyze the tacit knowledge explicating activity, we can see that the cognitive activity cannot be successful without any one of the factors in the functional system.

\section{Discussions}

Distributed cognition theory holds that cognition distributes in functional system, which is made up of individuals, artifacts, environment and culture. And tacit knowledge explicating activity is unexceptional, too. In a functional system, the change of any of these factors would cause the change of system. For example, the change of sharing tacit knowledge culture would cause the failure of tacit knowledge explicating, the usage of wrong technique tools would cause the failure, and the inappropriate information representation transform of others also would cause the failure, etc. The success of tacit knowledge explicating activity depends on interaction of each factor in a functional system. How to translate temporary unstable cognitive distributed system into stable distributed cognitive resource is an inspiration for us. For instance, to build up long-term resource pool and to cleanse and analyze the result of explicating can help us translate distributed cognition into stable distributed cognitive resource and help to explicate tacit knowledge effectively.

Factors in distributed system must depend on each other to accomplish one task, so none of the factors could be neglected. Communication is a necessary condition of distributed cognition, and shared information is pooled information, which can make someone who has the best resource apply the information for other's benefits [21]. In tacit knowledge explicating activity, sharing culture has important influence on the success of the activity, too. Only in sharing culture, each factor can communicate with each other effectively and the specific person can apply the useful information to cause the success of the explicating activity. So how to build up sharing culture in tacit knowledge explicating activity is a very important task.

The function of artifacts in the system is not only as tool, but also as a teacher sometimes. Artifacts expand and support human's intelligence, even they are more effective in some special task. When artifacts are applied, cognitive residue phenomenon will appear. When individual must finish some task without these artifacts, cognitive residue can provide efficient service for individual. For instance, the recorders can record those micro motions and effects of learners in detail. Even without the knowledge holder instruction and demonstration, the records could make learners go on to study. The cognitive residue phenomenon would support learners to understand the skill effectively. So in tacit knowledge explicating activity, how to choose and design suitable artifacts should also not be neglected. Such as, how to apply information bank and how to apply symbols, etc.

Individual is at the center of distributed cognitive system as cognitive subject [44]. The success of explicating activity is bound up with the cognitive structure of individual. If the specific person hasn't the corresponding professional knowledge, he couldn't succeed to explicate the knowledge. So for the purpose of sharing tacit knowledge effectively, the receiver with corresponding cognitive structure is an important factor, too.

Distributed cognition theory and study not only promote the development of cognitive theory, but also provide a good angle of view to study management problem, for instance, the study in team management [7]. At the same time, it has significance to study tacit knowledge explicating. However, many problems still need to be discussed more. For instance, how to design and choose suitable artifacts in different situation of tacit knowledge explicating, and how to discriminate suitable receivers to join the explicating activity, and how to build up suitable culture and environment in organization to push the success of tacit knowledge explicating activity, and so on.

\section{REFERENCES}

[1] W. M. Cohen and D. A. Leventhal, "Absorptive capacity: A new perspective on learning and innovation," Administrative Science Quarterly, Vol. 35, No. 1, pp. 128-52, 1990.

[2] M. Ipe, "Knowledge sharing in organizations: A conceptual framework," Human Resource Development Review, Vol. 2, No. 4, pp. 337-59, 2003. 
[3] R. W. Coff, D. C. Coff, and R. Eastvold, "The knowledgeleveraging paradox: How to achieve scale without making knowledge imitable," Academy of Management Review, Vol. 31, No. 2, pp. 452-465, 2006.

[4] I. Nonaka, "A dynamic theory of organizational knowledge creation," Organizational Science, Vol. 5, No. 1, pp. 14-37, 1994.

[5] Frappaolo, "Carl defining knowledge management: Four basic functions," Computerworld, 1998.

[6] R. L. Wang, X. M. Guo, and X. Q. Zheng, "The dimension and strategies of knowledge management," China Soft Science, Vol. 6, pp. 43-47, 2001.

[7] X. Wu and Z. M. Wu, "A study on team knowledge management based on shared mental model," R \& D Management, Vol. 18, No. 3, pp. 9-15, 2006.

[8] M. Polanyi, "Personal knowledge:Towards a post-critical philosophy," University of Chicago Press, Chicago, 1958.

[9] R. J. Sternberg, "Successful intelligence," Plume, New York, 1997.

[10] R. J. Sternberg, G. B. Forsythe, J. Hedlund, J. A. Horvath, R. K. Wagner, W. M. Williams, S. A. Snook, and E. L. Geigorenko, "Practical intelligence in everyday life," Cambridge University Press, New York, 2000.

[11] R. J. Sternberg and J. Hedlund, "Practice intelligence, g, and work psychology," Human Performance, Vol. 15, No. 1-2, pp. 143-160, 2002.

[12] P. F. Drucker, "The new productivity challenge," Harvard Business Review, 1991.

[13] C. T. Matthew and R. J. Sternberg, "Developing experience-based (tacit) knowledge through reflection," Learning and Individual Differences, Vol. 19, No. 4, pp. 530-540, December 2009.

[14] M. D. Koenig, "Knowledge Management," International Encyclopedia of Information and Library Science, 2nd Edition, In: J. Feather \& P. Sturges, Ed., Routledge, New York, pp. 351-359, 2003.

[15] R. E. Day, “Clearing up 'implicit knowledge': Implications for knowledge management, information science, psychology, and social epistemology," Journal of the American Society for Information Science and Technology, Vol. 56, No. 6, pp. 630-635, 2005.

[16] H. Taylor, "Tacit knowledge: Conceptualizations and operationalizations," International Journal of Knowledge Management, Vol. 3, No. 3, pp. 60-73, 2007.

[17] T. Gautschi, "The knowledge continuum," Design NewsAcademic Research Library, Vol. 54, No. 12, pp. 170, 1999.

[18] G. M. Zhou and X. L. Fu, "Distributed cognition: A new cognition perspective," Advance in Psychological Science, Vol. 10, No. 2, pp. 147-153, 2002.

[19] J. J. Gibson, "The ecological approach to visual perception," Houghton Mifflin, Boston, 1979.

[20] E. Hutchins, "Cognition in the wild," The MIT Press, 1995.

[21] P. Bell and W. Winn, "Distributed cognitions, by nature by design," East China Normal University Press, Shanghai, 2002.

[22] J. F. Ren and K. D. Li, "Distributed cognition theory and its application in CSCL system design," Audio-Visual Education Research, Vol. 136, No. 8, pp. 3-6, 2004.

[23] "Distributed cognitions: Psychological and educational considerations,” In: G. Salomon, Ed., Cambridge University Press, 1993.

[24] J. Chuah, J. Zhang, and T. R. Johnson, "Distributed cognition of a navigation instrument display task," In: M. Hahn and S. C. Stoness, Ed., Proceedings of the TwentyFirst Annual Conference of the Cognitive Science Society, Lawrence-Erbaumn, Mahwah, New Jersey, 1999.

[25] D. J. Teece, “Technology transfer by multinational Firms: The resource cost of transferring technological knowhow," The Economic Journal, Vol. 87, pp. 242-261, June 1977.

[26] W. Swap, D. Leonard, M. Shields, and L. Abrams, "Using mentoring and storytelling to transfer knowledge in the workplace," Journal of Management Information System, Vol. 18, No. 1, pp. 95-114, 2001.

[27] S. J. Hitt, "Tacit knowledge contained in internet/ web-based discussion group message," The Union Institute, pp. 36-37, 2001.

[28] K. U. Koskinen and H. Vanharanta, "The role of tacit knowledge in innovation process of small technology companies," International Journal of Production Economics, Vol. 80, pp. 57-64, 2002.

[29] S. Barnes, "Knowledge management system: Theory and practice," China Machine Press, Beijing, 2004.

[30] S. T. Zhang, T. Li, and X. M. Duan, "Tacit knowledge transferred model research in organization," Science Research Management, Vol. 25, No. 4, pp. 28-32, 2004.

[31] X. Y. Gao, "A model transformation of tacit knowledge based on ontology," Information Studies: Theory \& Application, Vol. 30, No. 1, pp. 41-45, 2007.

[32] Q. H. Liang and X. H. He, "Spatial clustering: The mechanism and path of tacit knowledge transferring and sharing," Management World, Vol. 3, pp. 146-147, 2006.

[33] J. S. Tang and J. S. He, "The knowledge fermenting models of organizational learning and individual learning," Scientific Management Research, Vol. 23, No. 1, pp. 86-88, 2005.

[34] C. Peng and H. B. Hu, "The mechanism of knowledge creation in knowledge alliance: BaS-C-SECI model," R\&D Management, Vol. 20, No. 1, pp. 118-122, 2008.

[35] Z. C. Gao and S. K. Tang, "The analysis of mechanism of enterprise knowledge creating based on cognitive psychology," Journal of Information, Vol. 8, pp. 87-91, 2008.

[36] J. X. Chu and S. K. Tang, "A Q-SECI model based on the insight learning and its application," Science Research Management, Vol. 28, No. 4, pp. 95-99, 2007.

[37] J. Z. Liu, "The inner mechanism of implicit cognition and tacit knowledge," Studies in Dialectics of Nature, Vol. 15, No. 6, pp. 11-14, 1999. 
[38] Z. Li and K. J. Zhang, "Tacit knowledge of cognitive structure," Social Sciences Journal of Hunan University, Vol. 4, pp. 38-41, 2007.

[39] B. Cronin, "Bowling alone together: Academic writing as distributed cognition," Journal of the American Society for Information Science and Technology, Vol. 55, No. 6, pp. 557-560, 2004.

[40] N. H. Schwartz, "Exploiting the use of technology to teach: The value of distributed cognition," Journal of Research on Technology in Education, Vol. 40, No. 3, pp. 389-404, 2008.

[41] F. Decortis, S. Noirfalise, and B. Saudelli, "Distributed cognition as framework for cooperative work [DB/OL]," 2005.
http://www-sv.cict.fr/cotcos/pjs/TheoreticalApproaches/ DistributedCog/DistCognition-paperDecortis.Htm.

[42] Y. Roger and J. Ellis, "Distributed cognition: An alternative framework for analyzing and explaining collaborative working," Journal of Information Tech- nology, Vol. 9, No. 2, pp. 119-128, 1994.

[43] Y. H. Yu, "Knowledge management and organizational innovation," Fudan University Press, Shanghai, 2001.

[44] D. N. Perkins, "Person-plus: A distributed view of thinking and learning," In: G. Salomon, Ed., Distributed Cognitions: Psychological and Educational Considerations," Cambridge University Press, 1993. 